\title{
Effet des conditions de traite sur la santé mammaire des vaches laitières et estimation des pertes en lait consécutives dans la région de Mahdia en Tunisie
}

\author{
Y. M'Sadak ${ }^{1 *}$ L. Mighri $^{2}$ K. Kraiem ${ }^{3}$
}

Mots-clés

Bovin - Vache laitière - Mammite Numération cellulaire somatique Hygiène du lait - Tunisie.

\begin{abstract}
Résumé
L'objectif de ce travail a consisté à évaluer la situation sanitaire mammaire des vaches à partir du taux cellulaire du troupeau (TCT) et du comptage cellulaire individuel $(\mathrm{CCl})$, et à estimer les pertes quantitatives en lait liées aux numérations cellulaires élevées. L'étude a été réalisée sur un échantillon de 30 élevages bovins de type hors-sol dans la région de Mahdia en Tunisie. Le diagnostic sanitaire mammaire a été effectué par la méthode de numération cellulaire directe sur lait de mélange et sur lait individuel. Les résultats ont montré que 87 p. 100 des élevages étudiés avaient des TCT supérieurs à 200000 cell./ml et 69 p. 100 des vaches avaient des CCI supérieurs à 200000 cell./ml. Les pertes quantitatives en lait consécutives à des taux cellulaires élevés ont été relativement importantes. Environ 23 p. 100 des élevages ont présenté des pertes en lait supérieures à 5 p. 100 de la production totale du troupeau et 15 p. 100 des vaches ont eu des pertes de lactation de 16 p. 100. Cette situation nécessiterait une intervention efficace de lutte contre les mammites, tout en essayant de maîtriser les facteurs de risque. L'étude des conditions de traite en relation avec les TCT et les CCI a montré que les pratiques de traite n'ont pas eu d'influence sur les taux cellulaires étudiés alors que les paramètres de fonctionnement de la machine à traire ont enregistré des corrélations significatives.
\end{abstract}

\section{INTRODUCTION}

En Tunisie, on estime qu'environ 30 p. 100 des vaches laitières sont réformées à cause de mammites (2). Cette situation a été accentuée avec le développement de l'élevage bovin laitier hors-sol et le recours à la mécanisation de la traite chez les petits éleveurs. Les mammites cliniques dont le dépistage visuel est facile posent un problème sérieux dans les élevages laitiers, alors que le dépistage des infections mammaires subcliniques nécessite le recours à des moyens de détection, tel que le comptage cellulaire dans le lait. Le présent travail a consisté à analyser la situation sanitaire mammaire chez les petits troupeaux bovins dans la région de Mahdia en

\footnotetext{
1. Equipement des fermes laitières, Institut supérieur agronomique de ChottMariem, BP 47-4042 Sousse, Tunisie.

2. Agriculture durable, Institut supérieur agronomique de Chott-Mariem, Tunisie. 3. Production bovine laitière, Institut supérieur agronomique de Chott-Mariem, Tunisie.

* Auteur pour la correspondance

Tél. : +21673327544; fax : 21673327591

E-mail : msadak.youssef@yahoo.fr
}

Tunisie, d'estimer les pertes en lait liées aux taux cellulaires élevés et de déterminer les facteurs de risque des mammites.

\section{MATERIEL ET METHODES}

\section{Enquête}

Une enquête a été conduite auprès de 30 élevages bovins laitiers dans la région de Mahdia. Les informations collectées ont concerné des données sur l'exploitant, l'exploitation, la conduite de l'élevage bovin laitier, le déroulement des chantiers de traite et le fonctionnement des machines à traire.

\section{Collecte d'échantillons de lait}

Des prélèvements d'échantillons du lait de mélange ont été effectués durant trois passages mensuels chez les 30 éleveurs. Les échantillons identifiés ont été mis dans des flacons en plastique contenant un conservateur (bichromate de potassium) et conservés au froid jusqu'au moment de l'analyse, sans dépasser la limite de 10 jours de conservation. Des prélèvements périodiques d'échantillons du lait individuel ont été effectués durant 17 mois et la conservation des échantillons s'est faite de la même manière. 


\section{Analyse des échantillons}

Les analyses des taux cellulaires du troupeau ont été effectuées par le compteur de cellules somatiques Anadis MI 600 au Laboratoire interprofessionnel d'analyse du lait (LIAL) à Sahline en Tunisie. Les analyses des comptages cellulaires individuels (CCI) ont été effectuées par un compteur cellulaire Fossomatic 4000 au laboratoire du service de contrôle laitier du Centre d'amélioration génétique de Sidi Thabet en Tunisie.

\section{Calcul des pertes en lait}

Les pertes quantitatives en lait liées aux taux cellulaires élevés ont été estimées à partir du modèle de troupeau établi par le Syndicat national des groupements techniques vétérinaires (Sngtv) français (13).

$\mathrm{P}(\%)=(\mathrm{TCT}-200000 / 100000) \times 0,02$

où $\mathrm{P}$ représente les pertes quantitatives en lait et TCT le taux cellulaire du troupeau (cell./ml).

\section{Analyses statistiques}

Les résultats des analyses de comptages cellulaires ont été traités par le logiciel SAS (2000) et les moyennes, les écarts-types et les fréquences ont été calculés.

\section{RESULTATS}

La répartition des TCT lors des trois passages dans les élevages est indiquée dans le tableau I. Les résultats des TCT enregistrés ont montré peu de variations entre les mois de prélèvement. La répartition des CCI dans les élevages ainsi que les pertes en lait consécutives sont présentées dans le tableau II.

L'application du modèle statistique du Sngtv sur les TCT des élevages suivis ont montré des pertes laitières variant entre 0 et 30 p. 100 de la production laitière totale des vaches. Le tableau III indique que 39 p. 100 des élevages suivis n'auraient pas eu des pertes en lait liées à l'élévation des TCT, et 23 p. 100 des élevages auraient eu une perte moyenne supérieure à 5 p. 100 de la production laitière. Entre les trois passages réalisés, la distribution des TCT dans les élevages selon les pourcentages de pertes était presque stable.

Les opérations de traite ainsi que leurs caractéristiques sont présentées dans le tableau IV. La majorité des élevages pratiquaient

TCT : taux cellulaire du troupeau l'hygiène et la rapidité étaient adoptées par environ la moitié des élevages.

Les résultats du testage des machines à traire sont montrés dans le tableau V. Par ailleurs, la fréquence de pulsation (FP) 55-60 puls./ min a été dans la norme pour 18 p. 100 des machines, alors que le rapport de pulsation (RP) 55-65 p. 100 l'a été pour 40 p. 100 des machines.

La recherche de l'impact des caractéristiques de traite sur les TCT et les CCI (tableau VI) n'a pas révélé d'influence significative des opérations de traite.

\section{Tableau I}

Répartition des TCT lors des trois passages dans les élevages

\begin{tabular}{lrrrr}
\hline TCT (cell./ml) & $\begin{array}{c}\text { TCT1 } \\
(\%)\end{array}$ & \multicolumn{1}{c}{$\begin{array}{c}\text { TCT2 } \\
(\%)\end{array}$} & $\begin{array}{c}\text { TCT3 } \\
(\mathbf{\%})\end{array}$ & $\begin{array}{c}\text { Total } \\
(\mathbf{\%})\end{array}$ \\
\hline$<200000$ & 30 & 40 & 40 & 13 \\
200000 à 500000 & 50 & 43 & 47 & 60 \\
500000 à 1000000 & 13 & 14 & 10 & 17 \\
$>1000000$ & 7 & 3 & 3 & 10 \\
\hline TCT moyen (cell./ml) & 374000 & 340000 & 346000 & 353000
\end{tabular}

TCT : taux cellulaire du troupeau

\section{Tableau II}

Répartition des $\mathrm{CCl}$ dans les élevages et pertes en lait consécutives

\begin{tabular}{lccc} 
CCI (cell. /ml) & $\begin{array}{c}\text { Vaches } \\
(\%)\end{array}$ & $\begin{array}{c}\text { Pertes/lactation } \\
(\%)\end{array}$ & $\begin{array}{c}\text { Pertes } \\
\text { (kg lait/lactation) }\end{array}$ \\
\hline$<200000$ & 31 & - & - \\
200000 à 500000 & 28 & 6 & 309 \\
500000 à 1000000 & 26 & 10 & 515 \\
$>1000000$ & 15 & 16 & 825
\end{tabular}

CCI : comptage cellulaire individuel

Tableau III

Estimation des pertes quantitatives en lait

\begin{tabular}{|c|c|c|c|c|c|c|c|c|}
\hline \multirow[t]{2}{*}{ Pertes (\%) } & \multicolumn{2}{|c|}{ TCT 1} & \multicolumn{2}{|c|}{ TCT 2} & \multicolumn{2}{|c|}{ TCT 3} & \multicolumn{2}{|c|}{ Total } \\
\hline & Effectif & $\%$ & Effectif & $\%$ & Effectif & $\%$ & Effectif & $\%$ \\
\hline 0 & 10 & 33 & 12 & 40 & 13 & 44 & 35 & 39 \\
\hline $0-5$ & 12 & 40 & 12 & 40 & 10 & 33 & 34 & 38 \\
\hline $5-10$ & 5 & 17 & 3 & 10 & 4 & 13 & 12 & 13 \\
\hline$>10$ & 3 & 10 & 3 & 10 & 3 & 10 & 9 & 10 \\
\hline Total & 30 & 100 & 30 & 100 & 30 & 100 & 90 & 100 \\
\hline
\end{tabular}


Concernant les paramètres de fonctionnement de la machine à traire, les FP et les RP ont eu une influence significative sur les CCI (tableau VII).

\section{DISCUSSION}

\section{Variation des numérations cellulaires}

Le TCT moyen enregistré a été de $353000 \pm 290000$ cell./ml. Cette moyenne a été élevée par rapport aux normes européennes (14) mais plus faible que celle trouvée par Mtaallah et coll. (10) sur un échantillon de grands troupeaux bovins laitiers au nord de la Tunisie $(626000 \pm 431000 \mathrm{cell} . / \mathrm{ml})$. D'autre part, les CCI pendant la période de 17 mois ont été en moyenne de 560000 $\pm 538000 \mathrm{cell} . / \mathrm{ml}$. Ces écarts-types élevés ont reflété une hétérogénéité marquée de la qualité cellulaire du lait dans l'échantillon étudié.

L'étude des TCT a montré que la probabilité d'infection des vaches par des mammites subcliniques (TCT > 500000 cell./ml) concernait 87 p. 100 des élevages. En outre, celle des CCI a montré que 28 p. 100 des vaches présentaient des mammites subcliniques (CCI entre 200000 et 500000 cell. $/ \mathrm{ml}$ ) et 41 p. 100 la possibilité de mammites cliniques $(\mathrm{CCI}>500000$ cell./ml).

\section{Estimation des pertes laitières}

Pour le calcul des pertes laitières, les auteurs ont choisi un modèle de troupeau simple et global qui tenait compte de la moyenne arithmétique des TCT (17). D'autre part, les modèles individuels sont dotés d'une grande précision et utilisent les numérations cellulaires individuelles avec une correction des pertes en fonction des paramètres individuels de variation $(1,5)$. Les pertes moyennes en lait estimées dans la présente étude représentaient 3,5 p. 100 de la production de lait, soit à une perte potentielle estimée à $1721 \mathrm{~kg}$ de lait/troupeau/an ou $215 \mathrm{~kg}$ de lait/vache/an.

\section{Tableau IV}

Opérations et caractéristiques de la traite des vaches

\begin{tabular}{lccc}
$\begin{array}{l}\text { Opérations de } \\
\text { traite contrôlée }\end{array}$ & $\begin{array}{c}\text { Eleveurs } \\
(\mathbf{\%})\end{array}$ & $\begin{array}{c}\text { Caractéristiques } \\
\text { de la traite }\end{array}$ & $\begin{array}{c}\text { Eleveurs } \\
(\mathbf{\%})\end{array}$ \\
\hline Lavage collectif des trayons & 93 & Traite hygiénique & 47 \\
Essuyage des trayons & 67 & Traite rapide & 53 \\
Elimination des premiers jets & 53 & Traite calme & 75 \\
Désinfection des trayons & 63 & Traite complète & 90
\end{tabular}

\section{Tableau V}

Paramètres de fonctionnement de la machine à traire

\begin{tabular}{lcccc} 
Paramètres & Norme & Conformité & < Norme & > Norme \\
\hline Vide de traite $(\mathrm{kPa})$ & $42-45$ & 21 & 54 & 25 \\
$\begin{array}{l}\text { Erreur indicateur } \\
\text { de vide (kPa) }\end{array}$ & Max. 2 & 21 & - & 79 \\
FP (puls/min) & $55-60$ & 18 & 28 & 54 \\
RP $(\%)$ & $55-65$ & 40 & 42 & 18
\end{tabular}

FP : fréquence de pulsation ; RP : rapport de pulsation
Pour une moyenne de lactation de $5158 \mathrm{~kg}$ de lait et en appliquant la relation entre les CCI et les pertes en lait établie par Radostitis et Blood en 1985, cités par Hanzen (7), 15 p. 100 des vaches contrôlées ont eu une moyenne des CCI supérieure à 1000000 cell./ml

\section{Tableau VI}

Recherche de l'impact des caractéristiques de traite sur les TCT et les CCI

\begin{tabular}{|c|c|c|}
\hline Caractéristiques & 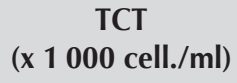 & 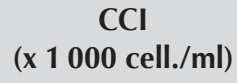 \\
\hline Lavage + essuyage & $285^{a}$ & $356^{\mathrm{a}}$ \\
\hline Lavage sans essuyage & $405^{a}$ & $577^{\mathrm{a}}$ \\
\hline Sans lavage ni essuyage & $496^{a}$ & $1088^{a}$ \\
\hline \multicolumn{3}{|l|}{ Contrôle $1^{\text {ers }}$ jets } \\
\hline Oui & $641^{\mathrm{a}}$ & $499^{a}$ \\
\hline Non & $659^{a}$ & $609^{a}$ \\
\hline \multicolumn{3}{|l|}{ Trempage } \\
\hline Oui & $584^{a}$ & $572^{a}$ \\
\hline Non & $762^{a}$ & $678^{a}$ \\
\hline $\begin{array}{l}\text { Contrôle } 1 \text { ers jets } \\
+ \text { trempage }\end{array}$ & $280^{a}$ & $152^{a}$ \\
\hline $\begin{array}{l}\text { Contrôle } 1^{\text {ers }} \text { jets } \\
\text { sans trempage }\end{array}$ & $641^{\mathrm{a}}$ & $609^{b}$ \\
\hline $\begin{array}{l}\text { Sans contrôle } 1^{\text {ers }} \text { jets } \\
\text { ni trempage }\end{array}$ & $762^{a}$ & $678^{b}$ \\
\hline \multicolumn{3}{|l|}{ Vide de traite } \\
\hline$<42 \mathrm{kPa}$ & $833^{a}$ & $801^{b}$ \\
\hline $42-45 \mathrm{kPa}$ & $465^{a}$ & $470^{a}$ \\
\hline$>45 \mathrm{kPa}$ & $639^{a}$ & $472^{a}$ \\
\hline \multicolumn{3}{|l|}{ Fréquence pulsation } \\
\hline$<55$ puls. $/ \mathrm{min}$ & $343^{a}$ & $502^{a}$ \\
\hline 55-60 puls./min & $441^{\mathrm{a}}$ & $381^{\mathrm{a}}$ \\
\hline$>60$ puls./min & $923^{b}$ & $745^{b}$ \\
\hline \multicolumn{3}{|l|}{ Rapport pulsation } \\
\hline$<55 \%$ & $393^{a}$ & $483^{a}$ \\
\hline $55-65 \%$ & $382^{a}$ & $436^{a}$ \\
\hline$>65 \%$ & $1699^{b}$ & $785^{b}$ \\
\hline
\end{tabular}

TCT : taux cellulaire du troupeau ; CCI : comptage cellulaire individuel

a,b Les différences entre les moyennes ont été statistiquement non significatives $(\mathrm{P}>0,05)$

\section{Tableau VII}

Coefficients de corrélation des paramètres de fonctionnement de la machine à traire avec les $\mathrm{CCl}$

\begin{tabular}{lccc} 
Variables & Vide de traite & FP & RP \\
\hline $\mathrm{CCl}$ & $-0,110^{\mathrm{ns}}$ & $0,118^{*}$ & $0,197^{* *}$
\end{tabular}

$\mathrm{CCI}$ : comptage cellulaire individuel

FP : fréquence de pulsation ; RP : rapport de pulsation

ns : corrélation non significative

* Corrélation significative $(\mathrm{P}<0,05)$; ** corrélation très significative $(\mathrm{P}<0,01)$ 
et les pertes consécutives ont été estimées à $825 \mathrm{~kg}$ de lait/vache/ lactation. Ces pertes en lait relativement importantes montrent la nécessité d'une assistance technique pour lutter contre les mammites subcliniques.

\section{Conditions de traite}

Le nettoyage et l'essuyage de la mamelle ont été effectués avec une lavette collective dans la quasi-totalité des élevages. Cette technique peut entraîner la transmission des germes responsables des mammites lors de la traite (11). L'étude statistique a révélé que l'absence de nettoyage et d'essuyage était associée à des TCT et des CCI élevés. Toutefois la différence entre les moyennes a été non significative $(\mathrm{P}>0,05)$. Ce résultat n'est pas semblable à celui trouvé par d'autres auteurs. En effet, en Tunisie Mtaallah et coll. (10), et en France Pluvinage et coll. (12) ont montré l'influence du nettoyage et de l'essuyage des trayons sur les TCT.

L'élimination des premiers jets avant la traite dans les élevages suivis se faisait généralement sur sol sous la vache, présentant ainsi un facteur de risque de contamination de la surface de couchage de la vache $(8,6)$. La seule technique utilisée pour la désinfection des trayons après la traite, dans la région d'étude, était le trempage des trayons dans une solution antiseptique. Cette désinfection des trayons n'était pas pratiquée quotidiennement. Elle était effectuée à la fin du chantier de traite et non pas immédiatement après la dépose des gobelets trayeurs.

La différence entre la moyenne des TCT des élevages qui pratiquaient l'élimination des premiers jets et le trempage, et celle des élevages qui ne les pratiquaient pas a été significative $(\mathrm{P}<0,05)$, et en accord avec les résultats de plusieurs auteurs $(10,15,16)$.

Concernant le fonctionnement de la machine à traire, un vide de traite faible augmente la durée de traite et peut être à l'origine d'une mauvaise traite ou de traite traumatisante (9). En effet, avec un vide de traite inférieur à $42 \mathrm{kPa}$, les TCT et les CCI enregistrés ont été élevés, mais la différence entre les trois classes de vide a été significative seulement avec les CCI.

La fréquence de pulsation a été élevée dans 54 p. 100 des machines, les éleveurs cherchant à l'augmenter pour diminuer la durée de la traite. Une pulsation défectueuse (> 60 puls./min) est en relation avec l'apparition de nouvelles infections et de lésions des trayons (9). Les moyennes des TCT et des CCI associées à une FP supérieure à 60 puls./min ont été plus élevées que les deux autres moyennes $(\mathrm{P}<0,05)$. Ces résultats sont semblables à ceux rapportés par Billon et coll. (4).

Le rapport de pulsation a été faible dans 42 p. 100 des machines, augmentant ainsi la durée de traite, avec aussi un risque de mauvaise traite. Le RP a été élevé dans 18 p. 100 d'entre elles entrầnant une traite défectueuse et un risque de mammite. Un RP entre 55 et 65 p. 100 a semblé être le meilleur compromis entre la rapidité de la traite et l'état sanitaire de la mamelle (3). Les moyennes des TCT et des CCI on été élevées $(\mathrm{P}<0,05)$ par rapport aux moyennes relatives des deux autres classes du RP.

\section{CONCLUSION}

Les conditions de traite des vaches ont été loin d'être respectées dans la majorité des élevages suivis. Les mauvaises conditions d'hygiène de la traite et le non-respect des paramètres de montage et de fonctionnement des machines à traire adoptées pourraient constituer des facteurs de risque de mammite. Cette étude a également montré que les paramètres de fonctionnement de la machine à traire avaient une influence sur les TCT et les CCI.

\section{Remerciements}

Ce travail, réalisé en Tunisie dans le cadre d'une action de recherche de l'Institution de la recherche et de l'enseignement supérieur agricoles (Iresa-Givlait), a été possible grâce à la contribution de la direction régionale de l'Office de l'élevage et des pâturages, du Groupement central des SMSA, de la SMSA Elhouda (Mahdia) et du laboratoire LIAL (Sahline).

\section{BIBLIOGRAPHIE}

1. BARTLETT P.C., MILLER G.Y., ANDERSON C.R., KIRK J.H., 1990. Milk production and somatic cell count in Michigan dairy herds. J. Dairy Sci., 73: 2794-2800.

2. BEN DHIAB H., 2002. Etude des mammites dans les petits élevages bovins de la région de Monastir. Tunis, Tunisia, PFE-INA, p. 54-75.

3. BILLON P., 2004. Machines à traire et mammites : comment interpréter les contrôles et les observations pour mieux conseiller les éleveurs ? In : Actes Journées nationales GTV, Tours, France, 29-31 mai 2002, p. 833-839.

4. BILLON P., SAUVEE O., MENARD J.L., GAUDIN V., 1998. Influence de la traite et de la machine à traire sur les numérations cellulaires et les infections mammaires chez la vache laitière. Renc. Rech. Ruminants, $\mathbf{5}$ : 305-312.

5. FABRE J.M., ROUSSE P., CONCORDET D., BERTHLOT X., 1990. Relation entre comptages cellulaires individuels et production en élevage bovin laitier dans le sud-ouest de la France ; analyse critique des méthodes statistiques utilisées. Revue Méd. vét., 141 : 361-368.

6. GUERIN P., GUERIN F., 2006. Les mammites de la vache laitière. Lyon, France, Ecole nationale de médecine vétérinaire, $140 \mathrm{p}$.

7. HANZEN C., 2009. Propédeutique de la glande mammaire, sémiologie et diagnostic individuel et de troupeau. Liège, Belgique, Université de Liège, p. 5-28.

8. LEVERSQUE P., 2004. L'observation des premiers jets. Prod. Lait québécois, 24.

9. MEZINE M., 2006. Analyse descriptive des facteurs de risque liés aux mammites dans des élevages d'une clientèle des Ardennes appliquant la démarche GTV Partenaire. Thèse Doct., Ecole nationale vétérinaire, Maisons-Alfort, France, $146 \mathrm{p}$.

10. MTAALLAH B., OUBEY Z., hAMMAMI H., 2002. Estimation des pertes de production en lait et des facteurs de risque des mammites subcliniques à partir des numérations cellulaires de lait de tank en élevage bovin laitier. Revue Méd. vét., 153 : 251-260.

11. NOIRETERRE P., 2006. Suivi de comptages cellulaires et d'examens bactériologiques lors de mammites cliniques chez la vache laitière. Thèse Doct., Ecole nationale vétérinaire, Lyon, France, 98 p.

12. PLUVINAGE P., DUCRUET T., JOSSE J., MONICAT F., 1991. Facteurs de risque des mammites des vaches laitières. Résultats d'enquête. Rec. Méd. vét., $167:$ 105-112.

13. RAGUET Y., 1996. Evaluation de l'impact économique des mammites. Bull. GTV, 3 B 527 : 21-24.

14. RAGUET Y., 1996. Qualité du lait - nouveaux services en élevages laitier. Résolution d'un problème complexe de cellules ( $2^{\mathrm{e}}$ partie). Bull. GTV, 4 B 528 : 5-42.

15. RASMUSSEN M.D., GALTON D.M., PETERSSON L.G., 1991. Effects of premilking teat preparation on spores anaerobes, bacteria, and iodine residues in milk. J. Dairy Sci., 74: 2472-2478.

16. SCHUKKEN Y.H., GROMMERS F.J., VAN DE GEER D., ERB H.N., BRAND A., 1991. Risk factors for clinical mastitis in herds with a low bulk milk somatic cell count. II. Risk factors for Escherichia coli and Staphylococcus aureus. J. Dairy Sci., 74: 826-832.

17. YALCIN C., STOTT A.W., LOGUE D.N., GUNN J., 1999. The economic impact of mastitis-control procedures used in Scottish dairy herds with high bulk-tank somatic-cell counts. Prev. vet. Med., 41: 135-149.

Accepté le 21.07.2011 


\section{Summary}

M'Sadak Y., Mighri L., Kraiem K. Effect of milking conditions on the udder health of dairy cows and ensuing milk loss estimation in the region of Mahdia in Tunisia

The aim of this study was to assess the health status of udders of cows based on the herd cell count (HCC) and the somatic cell count (SCC), and to estimate quantitative milk losses associated with high cell counts. The study was conducted on a sample of 30 landless cattle farms in Mahdia region in Tunisia. The udder health status was obtained by the direct cell count method in individual and mixed milks. Results showed that $87 \%$ of the studied farms had HCC higher than 200,000 cells/ $\mathrm{ml}$ and $69 \%$ of the cows had SCC higher than 200,000 cells/ $\mathrm{ml}$. Quantitative milk losses resulting from high cell rates were relatively high. About $23 \%$ of the farms showed milk losses higher than $5 \%$ of the herd total production and $15 \%$ of the cows presented $16 \%$ lactation losses. This situation would require efficient control against mastitis, while controlling risk factors. The study of milking conditions in relation to HCC and SCC showed that milking practices had no influence on the cell rates studied, whereas the functioning parameters of the milking machines showed significant correlations.

Keywords: Cattle - Dairy cow - Mastitis - Somatic cell count - Milk hygiene - Tunisia.

\section{Resumen}

M'Sadak Y., Mighri L., Kraiem K. Efecto de las condiciones de ordeño sobre la salud mamaria de las vacas lecheras y estimación de las pérdidas de leche consecuentes en la región de Mahdia en Túnez

El objetivo del presente trabajo consistió en evaluar la situación sanitaria mamaria de las vacas a partir de tasas celulares en el hato $(\mathrm{TCH})$ y de conteos celulares individuales $(\mathrm{CCl})$ así como estimar las pérdidas cuantitativas de leche relacionadas a los números celulares elevados. El estudio fue realizado con una muestra de 30 crías bovinas de tipo elevado en la región de Mahdia en Túnez. El diagnóstico sanitario mamario se efectuó mediante el método de numeración celular directa sobre la leche de mezcla y sobre la leche individual. Los resultados mostraron que $87 \%$ de las crías estudiadas presentaban TCH superiores a $200000 \mathrm{cel} / \mathrm{ml}$ y $69 \%$ de las vacas presentaban $\mathrm{CCl}$ superiores a $200000 \mathrm{cel} / \mathrm{ml}$. Las pérdidas cuantitativas en leche, consecuentes a las tasas celulares elevadas fueron relativamente importantes. Alrededor de $23 \%$ de los establecimientos presentaron pérdidas de leche superiores a $5 \%$ de la producción total del hato y de $15 \%$ de las vacas tuvieron pérdidas de lactación de 16\%. Esta situación necesitaría una intervención eficaz de lucha contra las mastitis, tratando al mismo tiempo de controlar los factores de riesgo. El estudio de las condiciones de ordeño en relación con las $\mathrm{TCH}$ y $\mathrm{CCl}$ mostraron que las prácticas de ordeño no tuvieron influencia sobre las tasas celulares estudiadas, mientras que los parámetros de funcionamiento de la máquina de ordeño registraron correlaciones significativas.

Palabras clave: Ganado bovino - Vaca lechera - Mastitis Conteo de células somáticas - Higiene de la lechera - Túnez. 